\title{
Power Allocation for In-Band Full-Duplex Self-Backhauling
}

\author{
Eva Lagunas, Lei Lei, Sina Maleki, Symeon Chatzinotas, Björn Ottersten \\ Interdisciplinary Centre for Security, Reliability and Trust (SnT), University of Luxembourg \\ Email: \{eva.lagunas,lei.lei,sina.maleki,symeon.chatzinotas,bjorn.ottersten\}@ uni.lu
}

\begin{abstract}
Ultra-dense cellular networks are emerging as one key characteristic of $5 \mathrm{G}$ wireless networks. In this context, frequency reuse is a fundamental technology enabler for efficient utilization of limited spectrum resources. In this paper, we investigate the in-band Full-Duplex (FD) self-backhauling architecture, in which Base Stations (BSs) operate on the same spectrum used by the users to access the network for backhauling purposes. In such a scenario, both cross-tier interference and self-interference due to the FD scheme at the BS should be taken into consideration. Apart from the frequency reuse, the network densification comes with the challenge of proper backhaul provisioning. In this paper, we propose a power allocation strategy for both the backhaul and access network such that the sum-rate is maximized and specifically tailored to tackle backhaul congestion.

Index Terms-Full-duplex; in-band; power allocation; selfbackhauling.
\end{abstract}

\section{INTRODUCTION}

Base Station (BS) densification has been proposed as an effective methodology to meet the requirements of the upcoming 5G wireless networks [1]. The upsurge in BS deployments would in turn require advanced backhauling infrastructure able to manage the expected high traffic flows. For ultradense deployment, in-band self-backhauling has emerged as an important enabler to balance cost in environments with scarce frequency resources [2]. In-band self-backhauling refers to the technique of leveraging the use of the spectrum utilized by the Radio Access Network (RAN) simultaneously for both the access and backhaul links.

In-band self-backhauling assumes the use of Full-Duplex (FD) technology at the BS in order to allow transmission and reception via the same spectrum [3]. FD has been recently considered as a viable technology thanks to the recent advances in antenna and circuit design [4]. Preliminary results have shown that FD has strong potential to increase the spectral efficiency of future wireless networks [5]. In our proposed scheme, we assume the BS to be equipped with a large antenna array able to do beam steering to serve different users at the same time. Beamforming not only mitigates the multi-user interference but it also reduces the self-interference, which is caused by leakage of the transmitted signal to the receiver chain.

In the considered scenario, mitigation of the interference between the access and the backhaul part of the network and

This work was partially supported by European Commission in the framework of the H2020 SANSA project (Grant agreement no. 645047) and by the National Research Fund, Luxembourg, under CORE project SeMIGod, SATSENT and FNR-FNRS bilateral project InWIP-NETs.

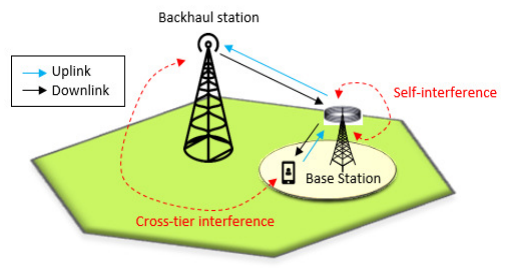

Fig. 1: Considered in-band self-backhauling scenario.

efficient allocation of transmitted power is of great significance. In particular, there are two types of interference to be considered, as depicted in Fig. 1: 1) Cross-tier interference caused by the transmission of the backhaul station and received at the user terminals, and 2) self-interference due to FD.

The interference between the backhaul and the access networks of in-band FD self-backhaul is a major limiting factor to the overall system throughput which can be alleviated using proper power allocation schemes [6]-[10].

In [6], a price-based resource allocation problem in a selfbackhauled small cell access point is investigated. Imperfect FD is considered but only the access links' transmitted powers are taken into account in the optimization problem. Moreover, scheduling is considered to serve the users.

The effect of FD on resource allocation in two-tier networks is studied in [7], and a solution is proposed for the power allocation problem that maximizes the downlink channel capacity. However, [7] considers a single cell network that provides access via a single BS together with a femtocell access point, both sharing the same spectral resources. A similar scenario is considered in [8], where a self-backhauling two-tier network consisting of one macro-BS with multiple antennas and BS with single antenna is considered, where both macro-BS and BS serve users simultaneously.

Although extensive research has been conducted in this area, none of them have focused on power allocation of both backhaul and access links, taking into account the interference associated with in-band FD self-backhauling together with the aggregate access traffic and the backhaul capacity limit. As highlighted in [11], backhaul has become a bottleneck for cellular systems, since most of the existing backhaul links have lower capacity and often cannot satisfy the aggregated rate requirements. In this context, the most relevant works are [9], [10]. Both [9], [10], consider the power allocation of a self-backhauling in-band FD access node with massive antenna arrays taking into account the backhaul capacity. 
While [9] focuses on maximization of user sum-rates, [10] considered minimization of transmit power subject to user rate requirements. However, the backhaul transmit power is assumed fixed and not considered in the power allocation problem.

Unlike [9], [10], in this paper we formulate the power allocation for both backhaul and access part of the network for an in-band FD self-backhauling scenario focusing on the downlink sum-rate maximizatiom. Moreover, we take into account the backhaul capacity so that power resources are not wasted to increase the access rate if the backhaul link capacity is not able to support the aggregate data rate.

The remainder of this paper is structured as follows. In Section II, the we introduce the system model formulation. In Section III, we formulate the power allocation optimization problem. In Section IV, we propose the a novel power allocation algorithm for both backhaul and access part. Finally, supporting numerical results are provided in Section $\mathrm{V}$, and Section VI states the conclusion.

\section{SySTEM MODEL}

We assume a BS equipped with an antenna array with capability to serve $K$ User Equipments (UEs) simultaneously via spatial multiplexing. The antenna array enables the implementation of spatial filtering which allows to significantly mitigate the multi-user interference [12]. Herein, the multiuser interference is assumed to be completely eliminated by the BS precoder. The BS is connected to the network via a single backhaul station, as depicted in Fig. 2. We assume a low-mobility environment where Channel State Information (CSI) of each UE is perfectly known at the BS. This condition enables the BS to dynamically allocate power per UE according to channel conditions.

The transmit power of the backhaul station is denoted by $P_{B}$ and the transmit powers of the BS are denoted by $p_{k}, k=$ $1, \ldots, K$, where the sub-index $k$ refers to a particular UE. Both the backhaul station and the BS are assumed to have its own power constraints, namely $P_{B} \leq P_{B}^{\max }$ and $\sum_{k=1}^{K} p_{k} \leq P_{A}^{\max }$, where $P_{B}^{\max }$ and $P_{A}^{\max }$ denote the maximum available power at the backhaul station and at the BS, respectively.

The received signal level at the BS receiver is determined by carrying out the analysis of the backhaul link and taking into account the self-interference due to the BS duplexing capability. To model the self-interference, let us consider the coefficient $0 \leq \kappa \leq 1$ as in [13], which reflects the BS capability in suppressing its own self-interference. The selfinterference power seen at the BS receiver is defined as follows:

$$
i_{s}=\kappa \sum_{k=1}^{K} p_{k}
$$

Therefore, when $\kappa=1$, no self-interference mitigation technique is used, while $\kappa=0$ models perfect self-interference cancellation. According to the previous discussion, the received signal level at the BS can be written as

$$
y_{B}=\sqrt{P_{B}} \gamma_{B} s_{B}+i_{s}+n_{B},
$$

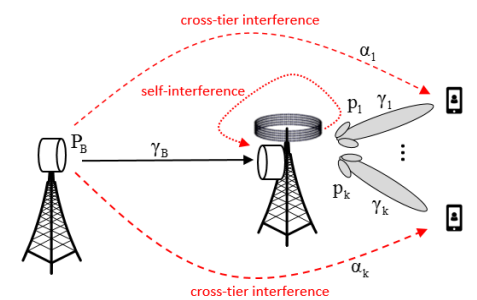

Fig. 2: System model

where $\gamma_{B}$ denotes the channel gain from the backhaul station to the BS, $s_{B}$ denotes the transmitted symbol with $\mathbb{E}\left\{\left|s_{B}\right|^{2}\right\}=$ 1 and $n_{B}$ is an additive noise modeled as a white zero-mean Gaussian noise with variance $\sigma_{B}^{2}$. Therefore, the backhaul link rate is given by

$$
R_{B}=\log _{2}\left(1+\frac{P_{B} \gamma_{B}}{\kappa \sum_{k=1}^{K} p_{k}+\sigma_{B}^{2}}\right) \quad[\text { bits/sec/Hz }] .
$$

Clearly, (3) shows the dependence of the backhaul link rate and the transmitted powers of both the backhaul $\left(P_{B}\right)$ and access networks, i.e. $p_{k}, k=1, \ldots, K$.

Regarding the access part of the network, the received signal at each of the UEs can be obtained analyzing the access link performance and tacking into account the interference coming from the backhaul transmission. Assuming an isotropic antenna at the UEs, the received signal at the $k$-th UE can be written as

$$
y_{k}=\sqrt{p_{k}} \gamma_{k} s_{k}+p_{B} \alpha_{k}+n_{k},
$$

where $\gamma_{k}$ denotes the channel gain between the BS and the $k$-th UE receiver, $\alpha_{k}$ denotes the interfering channel gain between the backhaul transmitter and the $k$-th UE receiver, $s_{k}$ denotes the symbols transmitted to the $k$-th user, with $\mathbb{E}\left\{\left|s_{k}\right|^{2}\right\}=1$, and $n_{k}$ is an additive noise modeled as a white zero-mean Gaussian noise with variance $\sigma_{k}^{2}$. Therefore, the rate of the $k$-th UE can be computed as follows

$$
R_{k}=\log _{2}\left(1+\frac{p_{k} \gamma_{k}}{P_{B} \alpha_{k}+\sigma_{k}^{2}}\right) \quad[\mathrm{bits} / \mathrm{sec} / \mathrm{Hz}] .
$$

Eq. (5) makes evident the coupling between the UEs' rates and the transmitted powers of both the backhaul $\left(P_{B}\right)$ and the corresponding access link $\left(p_{k}\right)$.

As mentioned before, the limitations of the backhaul link in terms of capacity support is one of the significant technical challenges faced by $5 \mathrm{G}$ networks. In this paper, we make use of power allocation to ensure that the traffic in the access link is always lower than or equal to the traffic supported by the fronthaul link. In a mathematical notation this translates into the following

$$
\sum_{k=1}^{K} R_{k} \leq R_{B}
$$

Therefore, the main goal of this paper is to optimally allocate the powers, namely $P_{B}$ and $p_{k}$, for $k=1, \ldots, K$, so that the sum-rate of the access links is maximized while respecting the capacity limits of the backhaul link. 


\section{Problem Formulation}

The power allocation optimization problem can be formulated as maximization of the sum of the user rates, defined as $R_{A}=\sum_{k=1}^{K} R_{k}$, which is one of the most popular figures of merit for measuring the performance of a communication system. Denoting $P_{A}=\sum_{k=1}^{K} p_{k}$, the power allocation problem is given in (7).

$$
\begin{array}{|ll}
\hline \max _{P_{B}, p_{k}} & R_{A} \\
\text { s.t. } & R_{A} \leq R_{B} \\
& P_{B} \leq P_{B}^{\max } \\
& P_{A} \leq P_{A}^{\max } \\
\hline
\end{array}
$$

Given the non-linear coupling between the optimization variables in the utility function of (7), the problem is intractable. This is because both the transmit power of the backhauling station and the transmit power of the BS directly affect the sum-rate of the user rates. Hence, computing the optimal solution to (7) is very challenging. Therefore, we propose an algorithm to solve this problem iteratively.

\section{Proposed Power Allocation Algorithm}

To solve (7), in this paper we make use of decomposition methods in which the coupled problem is split into smaller sub-problems. More precisely, in this paper we will solve (7) by iteratively solving the access and the backhaul part of the network by considering the coupled interference as noise. Clearly, maximization of information rate under (fixed) Gaussian noise channel conditions translate into maximization of transmitted power. Therefore, the proposed algorithm starts assigning maximum transmit power to both backhaul station and BS. Next, the algorithm gradually reduces the transmit powers until the difference between $\sum_{k=1}^{K} R_{k}$ and $R_{B}$ is minimal.

The proposed algorithm for the power assignment is summarized in Algorithm 1, which makes use of the previous ideas. Essentially, the algorithm consists of iteratively solving the power allocation of the access network, regarding the backhaul power allocation as fixed. The latter is done using the well-known water-filling power allocation, which maximizes the information rates in the access links by regarding the backhaul interference as noise:

$$
p_{k}= \begin{cases}\frac{1}{\nu}-\beta_{k}, & \text { if } \nu<\frac{1}{\beta_{k}} \\ 0, & \text { otherwise }\end{cases}
$$

where $\beta_{k}=\frac{\sigma_{k}^{2}+P_{B} \alpha_{k}}{\gamma_{k}}$ act as an indicator of the $k$-th UE channel condition.

Once the access powers are obtained, both the access rate and the backhaul rate can be computed. If the backhaul rate $\left(R_{B}\right)$ is higher than the access rate $\left(R_{A}\right)$ it means that we are putting some power to the backhaul station that is not required. Therefore, the backhaul power $\left(P_{B}\right)$ should be reduced. On the other hand, if the access rate is higher than the backhaul rate, it means that the backhaul cannot support the amount of traffic generated by the UEs and, thus, BS should reduce

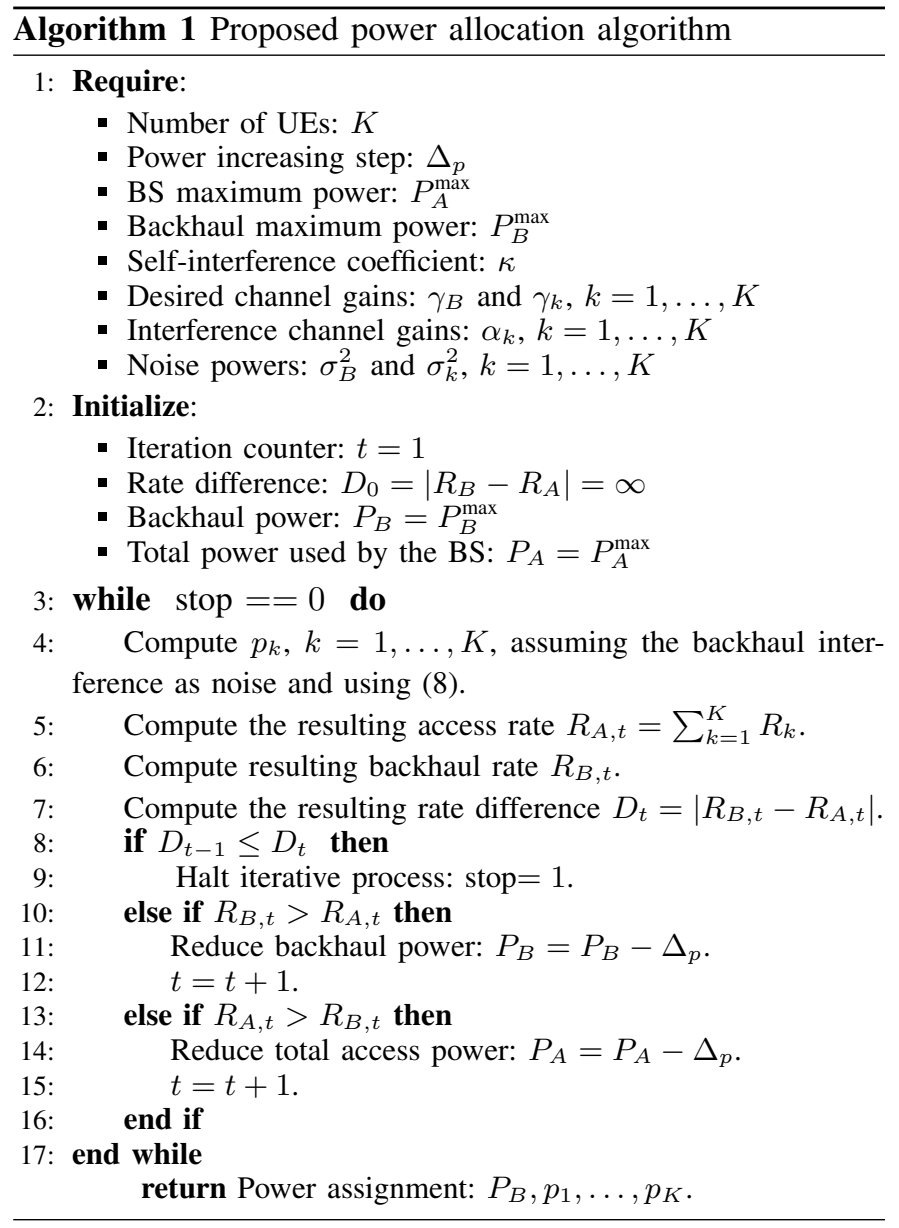

its transmit power $\left(P_{A}\right)$. Inspired by the conventional gradient descent methods [14], we make use of a power step for both the backhaul station and the BS, which is denoted herein by $\Delta_{p}$. The power step $\Delta_{p}$ should be small enough in order to achieve convergence and high enough to speed up the convergence. Eventually, the algorithm converges since the difference between $D=\left|R_{B}-R_{A}\right|$ decreases until it hits a local optimum point.

\section{Simulation Results}

In this section, we make use of numerical results to evaluate the proposed power allocation solution. Figure 3 illustrates the set of all possible rate solutions $\left(R_{A}\right.$ and $\left.R_{B}\right)$ resulting from (7), which is typically referred in the literature as the Pareto feasible set. Figure 3 considers $K=64, P_{A}^{\max }=P_{B}^{\max }=10$ $\mathrm{W}, \Delta_{p}=0.05 \mathrm{~W}$, and $\sigma_{B}^{2}=\sigma_{k}^{2}=1$, for $k=1, \ldots, K$. The channel gains $\alpha_{k}$ and $\gamma_{k}, k=1, \ldots, K$, are generated from a uniform random distribution within $[\bar{\alpha}-0.1, \bar{\alpha}+0.1]$ and $[\bar{\gamma}-0.1, \bar{\gamma}+0.1]$, where $\bar{\alpha}$ and $\bar{\gamma}$ denote the mean of the uniform distribution. In Figure 3, we evaluated three different scenarios: (a) Low self-interference $(\kappa=0.2)$ and good user conditions $(\bar{\alpha}=0.9$ and $\bar{\gamma}=0.3)$; (b) High selfinterference $(\kappa=0.8)$ and good user conditions $(\bar{\alpha}=0.9$ and $\bar{\gamma}=0.3)$; and (c) Low self-interference $(\kappa=0.2)$ and bad user conditions $(\bar{\alpha}=0.5$ and $\bar{\gamma}=0.6)$. In the first two cases, the starting point does not satisfy the constraint $R_{A} \leq R_{B}$ and, thus, the algorithm reduced the BS power until it reaches 


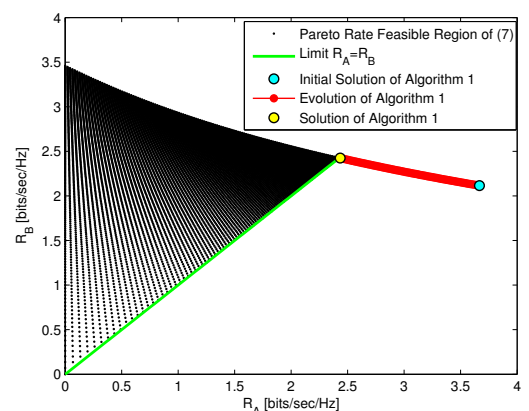

(a)

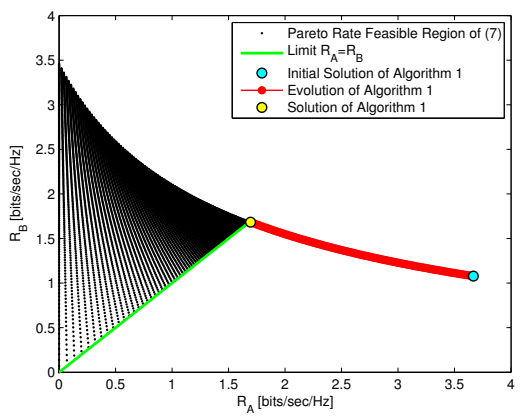

(b)

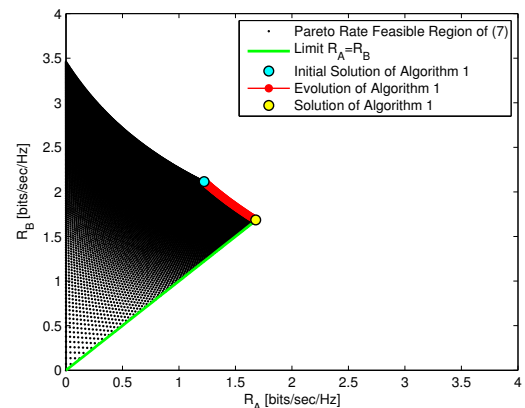

(c)

Fig. 3: Feasible set and proposed power allocation for $K=5, P_{A}^{\max }=P_{B}^{\max }=10 \mathrm{~W}$ and $\Delta_{p}=0.05 \mathrm{~W}:(\mathrm{a}) \bar{\gamma}=0.9, \bar{\alpha}=0.3$ and $\kappa=0.2$, (b) $\bar{\gamma}=0.9, \bar{\alpha}=0.3$ and $\kappa=0.8$, and (c) $\bar{\gamma}=0.6, \bar{\alpha}=0.5$ and $\kappa=0.2$.

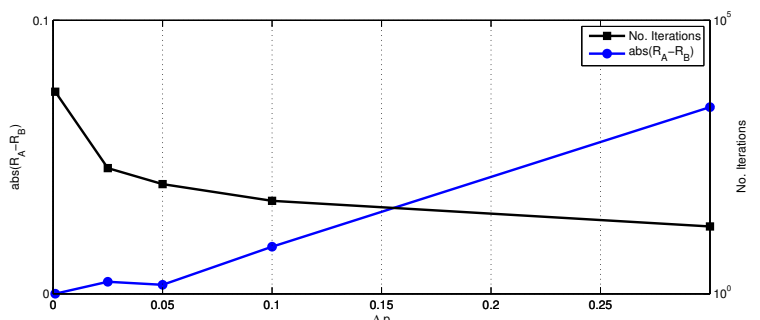

Fig. 4: Effect of $\Delta p$ in the speed and accuracy of the proposed algorithm.

the equality point $R_{A}=R_{B}$. The last case, illustrated in Fig. 3(c), the starting point satisfies $R_{A} \leq R_{B}$ but the backhaul capacity is overdimensioned compared to the access capacity. Therefore, the algorithm reduced the backhaul power so that the access network conditions are improved until reaching the equality point $R_{A}=R_{B}$. In all case, the proposed algorithm converges to the optimal point.

In Fig. 4, we show how the step size $\Delta p$ affect the convergence speed of the algorithm as well as the steady state error of the algorithm's solution. As expected, the higher the step-size, the faster the convergence to the optimal point. On the other hand, the accuracy of the algorithm's solution improves by using lower values of $\Delta p$. Note that fluctuations around the optimal point are avoided with the stoping criteria proposed in Algorithm 1.

\section{CONCLUSION}

In this paper, we investigated the power allocation problem for the proposed in-band self-backhaul scheme with multiple antennas and FD at the BS, which enables the access and backhauling simultaneously in the same frequency band. Unlike previous works on this subject, we optimize the BS transmit powers together with the backhaul station transmit power, which in turn dictates the backhaul capacity limit to support the aggregate user rate. An iterative algorithm has been proposed which successfully allocates the powers in the considered scenario so that the users' sum-rate is maximized tacking into account the capacity limits of the backhaul link. In our future work, we plan to extend the proposed formulation for the case where the beamforming at the access part is not perfect and, thus, introduces residual interference.

\section{REFERENCES}

[1] A. Gupta and R.K. Jha, "A Survey of 5G Network: Architecture and Emerging Technologies," IEEE Access, vol. 3, pp. 1206-1232, Aug. 2015.

[2] A. Sabharwal, P. Schniter, D. Guo, D.W. Bliss, S. Rangarajan, and R. Wichman, "In-Band Full-Duplex Wireless: Challenges and Opportunities," IEEE J. Select. Areas Commun., vol. 32, no. 9, pp. 1637-1652, Sep. 2014.

[3] R.A. Pitaval, O. Tirkkonen, R. Wichman, K. Pajukoski, E. Lahetkangas, and E. Tiirola, "Full-Duplex Self-Backhauling for Small-Cell 5G Networks," IEEE Wireless Commun., vol. 22, no. 5, pp. 83-89, Oct. 2015.

[4] G. Liu, F.R. Yu, H. Ji, V.C.M. Leung, and X. Li, "In-Band Full-Duplex Relaying: A Survey, Research Issues and Challenges," IEEE Commun. Surveys Tuts., vol. 17, no. 2, pp. 500-524, May 2015.

[5] M. Duarte and A. Sabharwal, "Full-Duplex Wireless Communications Using Off-the-Shelf Radios: Feasibility and First Results," Asilomar Conf. Signals, Syst. Comput., Pacific Grove, CA, USA, pp. 1558-1562, Nov. 2010.

[6] A. Rahmati, A. Sadeghi, and V. Shah-Mansouri, "Price-Based Resource Allocation for Full Duplex Self-Backhauled Small Cell Networks," IEEE ICC, London, UK, Jun. 2015.

[7] R. Sultan, L. Song, and Z. Han, "Impact of Full Duplex on Resource Allocation for Small Cell Networks," IEEE GlobalSIP, Atlanta, GA, USA, pp. 1257-1261, Dec. 2014.

[8] L. Chen, F.R. Yu, H. Ji, V.C.M. Leung, X. Li, and B. Rong, “A FullDuplex Self-Backhaul Scheme for Small Cell Networks with Massive MIMO," IEEE ICC, Kuala Lumpur, Malaysia, 2016.

[9] D. Korpi, Riihonen T., and M. Valkama, "Self-Backhauling Full-Duplex Access Node with Massive Antenna Arrays: Power Allocation and Achievable Sum-Rate," EUSIPCO, Budapest, Hungary, pp. 1618-1622, Aug. 2016.

[10] D. Korpi, T. Riihonen, and M. Valkama, "Inband full-duplex radio access system with self-backhauling: Transmit power minimization under QoS requirements," IEEE ICASSP, New Orleans, LA, USA, Mar. 2017.

[11] H.S. Dhillon and G. Caire, "Wireless Backhaul Networks: Capacity Bounds, Scalability Analysis and Design Guidelines," IEEE Trans. Wireless Commun., vol. 14, no. 11, pp. 6043-6056, Jun. 2015.

[12] A. Alkhateeb, G. Leus, and R.W. Heath, "Limited Feedback Hybrid Precoding for Multi-User Millimeter Wave Systems," IEEE Trans. Wireless Commun., vol. 14, no. 11, pp. 6481-6494, Nov. 2015.

[13] N. Pappas, M. Kountouris, A. Ephremides, and A. Traganitis, "RelayAssisted Multiple Access With Full-Duplex Multi-Packet Reception,' IEEE Trans. Wir. Commun., vol. 14, no. 7, pp. 3544-3558, Jul. 2015.

[14] S. Boyd and L. Vandenberghe, Convex Optimization, Cambridge University Press, Cambridge, UK, 2004. 